\title{
Simulación computacional de un ensayo Erichsen aplicado a una chapa de acero DC-06
}

\section{Computational simulation of an Erichsen test applied on a DC-06 steel sheet}

\author{
Nazareno Antúnez ${ }^{1}$, Vitaliy Martynenko ${ }^{1}$ Germán Abate ${ }^{1}$, \\ Daniela Perez ${ }^{1}$, Wadi Chiapparoli ${ }^{2}$, \\ Daniel Martínez Krahmer ${ }^{1}$
}

\footnotetext{
${ }^{1}$ Centro de Investigación y Desarrollo en Mecánica, Instituto Nacional de Tecnología Industrial, Avpreenida General Paz 5445, (1650) Miguelete, Provincia de Buenos Aires, Argentina.

e-mail: nantunez@inti.gob.ar; vmart@inti.gob.ar; gabate@inti.gob.ar; danielap@inti.gob.ar; mkrahmer@inti.gob.ar

${ }^{2}$ Instituto Argentino de Siderurgia, Avenida Central 1975, (2900) San Nicolás, Provincia de Buenos Aires, Argentina. e-mail: chiapparoli@siderurgia.org.ar
}

\section{RESUMEN}

En la actualidad, la utilización de softwares de simulación computacional aplicados a distintos procesos de conformado, como puede ser el estampado y sus variantes, forma parte habitual de las actividades de desarrollo de las grandes industrias, y también comienzan a introducirse lentamente dentro de las PyMEs. Una de sus principales ventajas radica en la reducción significativa de los costos de desarrollo al afrontar nuevos proyectos, tanto de nuevos productos como de procesos. Asimismo, estas herramientas son ampliamente usadas para reproducir fenómenos físicos complejos.

Durante un proceso de embutido (estampado profundo de chapas), el material es fuertemente solicitado, razón por la cual se requiere de un conocimiento preciso de las propiedades del mismo, como ser la ductilidad, resistencia mecánica y plasticidad. Es por ello que, se realizan ensayos de caracterización, como puede ser el ensayo Erichsen, durante el cual se recrea de manera controlada un proceso de estirado.

En el presente trabajo, se efectuó la simulación de un ensayo de embutibilidad empleando el software Simufact.Forming, aplicado a una chapa de acero DC06-base IF de 0,7 mm de espesor nominal. Durante la simulación se consideraron las condiciones de lubricación, la presión del prensachapa, las dimensiones de las matrices y el coeficiente de rozamiento. Al mismo tiempo, se efectuó el ensayo experimental, con el objetivo de comparar los resultados obtenidos y validar los resultados simulados.

Finalmente, se compararon las cargas de embutido, las geometrías de las copas, y el perfil de espesores resultantes en una sección diametral de la misma.

Palabras Clave: Ensayo Erichsen, estampado profundo, simulación, chapa.

\begin{abstract}
Nowadays, the use of computer simulation on forming processes as stamping and its variants, is an habitual development activity of big industries, and also it is slowly being introduced in SME (Small and Mediumsize Enterprise). One of the main advantages of it is application lies-in the reduction of costs regarding new projects, products and processes developments. Likewise, these tools are widely used to reproduce complex physical phenomena.

During the deep drawing process, the material is mechanically stressed, therefore requires knowledge of it is properties, such as ductility, mechanical strength and plasticity. Thus, characterization tests like the Erichsen test are performed to recreate a controlled deep drawing process.

In this paper, using the Simufact.Forming software, a deep drawing test was simulated, for a steel sheet DC06 IF-base, of nominal thickness $0.7 \mathrm{~mm}$. During the simulation is taken into account the conditions of lubrication, pressure of blank holder, dimensions of the dies and friction coefficient. At the same time, the
\end{abstract}


experimental test was also performed in order to compare and validate the simulated results.

Finally, loads of deep drawing, the geometries of the cups, and the diametral section thickness of the steel sheet, were compared.

Keywords: Erichsen test, deep drawing, simulation, sheet.

\section{INTRODUCCIÓN}

Existe hoy en día y a nivel mundial, una gran difusión de herramientas de simulación computacional. Estos softwares permiten trabajar en forma colaborativa con los procesos de fabricación existentes, teniendo incluso la capacidad de considerar las diferentes variables que intervienen en los mismos.

Sin embargo, en Argentina, el desarrollo de un proceso de estampado de chapa se basa principalmente en los conocimientos intrínsecos de los matriceros y operarios de máquina. Esta forma de trabajo no se asocia al concepto de colaboración tecnológica como ocurre al usar métodos de simulación numérica, y esto podría explicarse por el costo de adquisición del software y la necesidad de contar con operadores de alta especialización.

Una ventaja radical de estas herramientas reside en que, una vez adquirida y efectuado el aprendizaje correspondiente, brindan grandes reducciones de costos por optimización de los procesos, disminución del material de descarte, y minimización de los tiempos de diseño.

Si bien en la actualidad su empleo no se limita a obtener las dimensiones ideales de matrices y seleccionar las máquinas correctas según sea el proceso y el material de trabajo, una revisión del estado del arte nos muestra que están comenzando a usarse en forma inversa, esto es, para obtener las propiedades del material. Esto resulta así en los trabajos de Gavrus y Oleksik [1,2] que lo hicieron en base a simular el ensayo Erichsen.

Si bien existen diversas investigaciones publicadas que muestran la utilidad de los softwares de simulación, y que uno de los trabajos esenciales consiste en contrastar experimentalmente los resultados obtenidos en forma numérica, en relación al ensayo Erichsen, hay pocos antecedentes. Mientras Akrout [3] comparó valores del índice Erichsen y de la carga de conformado, obtenidos en forma experimental y por simulación 2D, sobre chapas de diversos materiales y un rango de espesor comprendido entre $1 \mathrm{~mm}$ a $2 \mathrm{~mm}$, con y sin uso de lubricante, Esmaeilizadeh [4] trabajó sobre chapas de una aleación de aluminio AA1200 de $2 \mathrm{~mm}$ de espesor usando simulación computacional en 3D, con el objeto de obtener la carga de conformado y el grado de deformación. Incluso, este último lo hizo considerando y sin tener en cuenta las condiciones de anisotropía del material, verificando un mejor ajuste con los valores experimentales en el primer caso.

En razón de lo expuesto, hemos considerado trabajar con una chapa fina de acero IF DC06 de 0,7 mm de espesor, realizando una comparación de los valores de carga y de variación de espesor en la copa, obtenidos en forma experimental y por simulación en 3D, considerando durante la simulación la anisotropía de la chapa, las condiciones de lubricación, la presión del prensachapa, la dirección de laminación, la temperatura, y la recuperación elástica.

\section{MATERIALES Y MÉTODOS}

El desarrollo del trabajo se va a dividir en dos secciones, la primera, correspondiente a la caracterización de la realización del ensayo, y la segunda, al proceso de simulación, que incluye la obtención de los valores numéricos de los parámetros que utiliza el software.

El ensayo Erichsen fue realizado sobre una chapa de $0.7 \mathrm{~mm}$ de espesor nominal, de un acero tipo DC06 base IF, es decir, un material con alta capacidad de deformación, usado en el estampado de diferentes piezas en la industria.

A continuación, se adjuntan tablas con las características físicas, químicas y mecánicas de la chapa, siendo la composición química obtenida a partir de bibliografía indicándose en la tabla 1 los valores máximos de cada componente según la norma UNE-EN 10130:2008 y las características físicas y mecánicas las obtenidas a partir de un ensayo de tracción realizado por una empresa privada sobre el material de la chapa. Dicho ensayo fue realizado en una máquina de ensayos Zwick/Roell dentro del marco de la norma Euronorm para ensayos de tracción. 
Tabla 1: Composición química del acero DC-06 base IF [5]

\begin{tabular}{l|l|l|l|l}
\hline ELEMENTO & C & MN & P & S \\
\hline Cantidad (\% máx.) & 0,02 & 0,25 & 0,02 & 0,02 \\
\hline
\end{tabular}

Tabla 2: Características físicas y mecánicas de la chapa ensayada

\begin{tabular}{|c|c|c|c|c|c|c|c|c|c|}
\hline \multirow[t]{2}{*}{ PRODUCTO } & \multirow[t]{2}{*}{ MATERIAL } & \multirow{2}{*}{$\begin{array}{l}\text { TENSIÓN } \\
\text { DE } \\
\text { FLUENCIA } \\
(\mathrm{MPa}) \\
\end{array}$} & \multirow{2}{*}{$\begin{array}{l}\text { TENSIÓN } \\
\text { DE RO- } \\
\text { TURA } \\
\text { (MPa) }\end{array}$} & \multirow{2}{*}{$\begin{array}{l}\text { ALARGAMIENTO } \\
(\%)\end{array}$} & \multirow{2}{*}{$\begin{array}{l}\text { ENDURECIMIENTO } \\
\text { POR DEFORMA- } \\
\text { CIÓN }\end{array}$} & \multicolumn{4}{|c|}{ ANISOTROPÍA } \\
\hline & & & & & & $\mathrm{RO}^{\circ}$ & $R 45^{\circ}$ & R90 ${ }^{\circ}$ & $\begin{array}{l}\text { R ME- } \\
\text { DIO }\end{array}$ \\
\hline Chapa & $\begin{array}{l}\text { DC06- } \\
\text { Base IF }\end{array}$ & $129-132$ & 285-291 & $47-49$ & 0,24 & 1,68 & 1,75 & 2,17 & 1,84 \\
\hline
\end{tabular}

El ensayo Erichsen se desarrolló obedeciendo a un marco general que a continuación se describirá.

En cuanto a la geometría y dimensiones de las partes involucradas en el ensayo, se pueden apreciar en el siguiente gráfico:

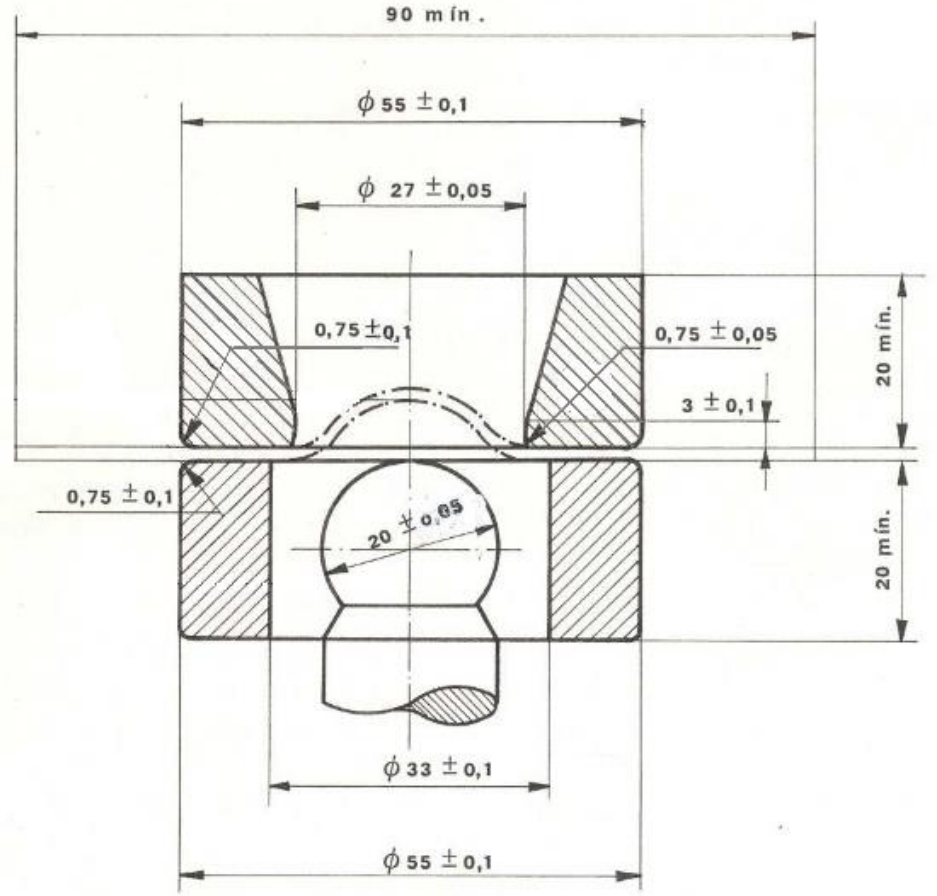

Figura 1: Dimensiones de los elementos utilizados en el ensayo Erichsen

El desarrollo del ensayo se realizó de la siguiente manera. Primeramente, se coloca la probeta dentro de la máquina, en este caso una chapa rectangular de $90 \mathrm{~mm}$ de ancho por $170 \mathrm{~mm}$ de longitud aproximadamente. Una vez ubicada debe ser asegurada por el prensachapa. Seguidamente comienza el descenso del punzón de 20mm de diámetro a una velocidad de $2 \mathrm{~mm} / \mathrm{s}$. Una vez ocurrido el contacto entre el punzón y la chapa, la distancia recorrida por el primero fue de $9 \mathrm{~mm}$. 


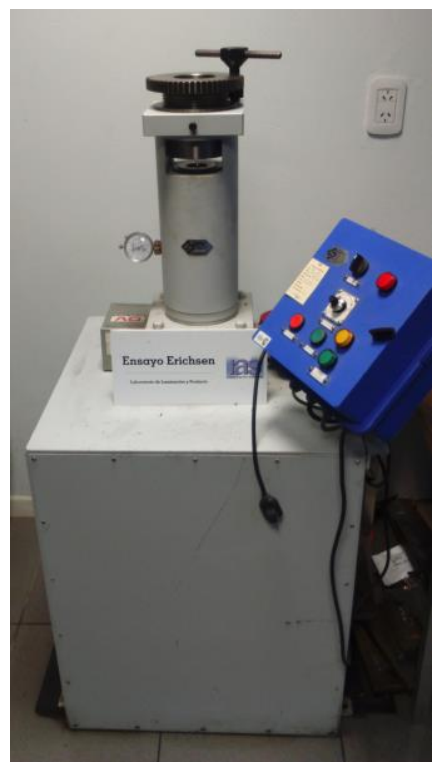

Figura 2: Equipo de laboratorio utilizado para la realización del ensayo Erichsen

En la figura 3 se observa la zona deformada de la chapa luego del ensayo, la fuerza máxima medida en el punzón fue de $1250 \mathrm{~kg}$. En dicha imagen se indica la dirección de laminación. Para establecer la variación de espesor, se cortó la chapa por el centro de la copa, en forma transversal a dirección de laminación, empleando electroerosión por hilo.

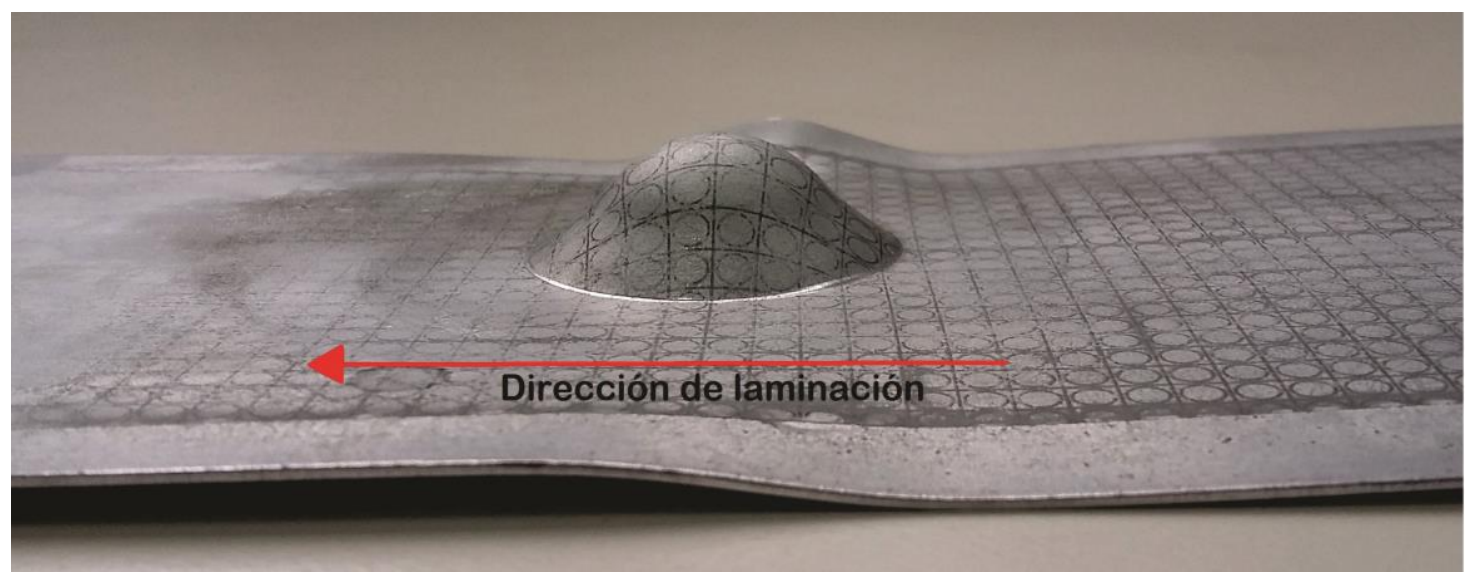

Figura 3: Deformación en forma de copa en la chapa ensayada. Junto con ésta se observa la dirección de laminación.

Utilizando un banco de medición óptico Dormer de apreciación centesimal, se midieron tres zonas específicas sobre la copa previamente cortada, obteniendo así el espesor de la chapa en la altura máxima de la copa, en la altura media y en el sector del espesor mínimo. Los valores medidos se muestran en la tabla 3:

Tabla 3: Resultados de las mediciones de espesor de la copa Erichsen

\begin{tabular}{|l|l|l|l|l|}
\hline ZONA & ALTURA MÁXIMA & ALTURA MEDIA & ESPESOR MINIMO & ESPESOR INICIAL \\
\hline ESPESOR & 0.59 & 0.58 & 0.55 & 0.71 \\
\hline
\end{tabular}

Una vez relevados los espesores de la copa, se comenzó el trabajo de simulación. Se modeló mediante un software de tipo CAD, las geometrías de los elementos para la realización del ensayo, que luego fueron importados en el Simufact.Forming.

Con el fin de disminuir el tiempo de simulación, se analizó solamente un cuarto de la chapa estableciendo los planos de simetría correspondientes. 


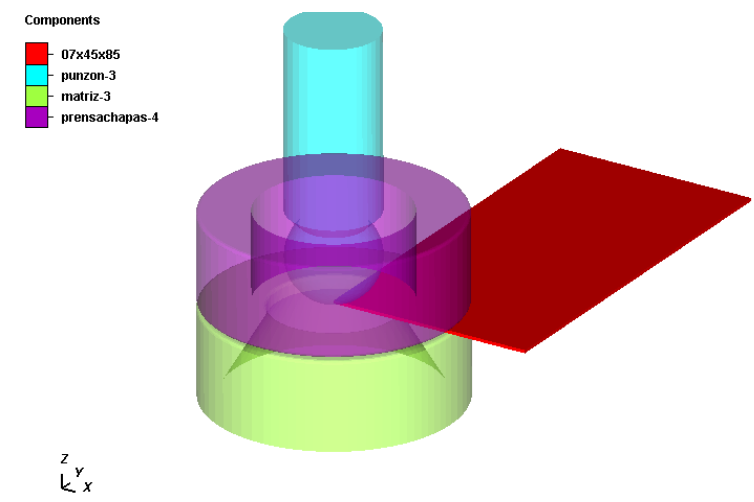

Figura 4: Cuerpos geométricos importados en el Simufact y 1/4 de la chapa a ensayar

Para realizar la simulación se utilizó un acero incluido en la librería del software similar en su composición química al material de la chapa ensayada, dentro de la biblioteca ya tiene parametrizados los valores de propiedades mecánicas, térmicas y curvas de fluencia siendo regido por la ley de plasticidad de Hill. Conociendo que el software permite modificar estas propiedades en los materiales que tiene cargados por defecto, para que exista representatividad entre el material ensayado y el simulado, a este último se le agregaron las propiedades anisotrópicas y mecánicas del DC06 base IF presentes en la tabla 2. Una vez establecidas la geometría y su material, fue necesario también indicar la dirección de laminación siendo ésta paralela al eje Y, a fin de establecer un punto de relación con la pieza, de los valores de anisotropía.

En cuanto a las temperaturas de ambiente y matriz, ambas fueron $20^{\circ} \mathrm{C}$ ya que el ensayo se realizó a temperatura ambiente.

Se asignó también la presión del prensachapa, siendo la misma de $1.1 \mathrm{~N} / \mathrm{mm}^{2}$. Por otro lado, en relación al ensayo realizado, la carrera del punzón fue establecida en $9 \mathrm{~mm}$.

Un dato de entrada a desarrollar fue el coeficiente de rozamiento entre el punzón y la chapa, ya que solo se conocía el tipo de lubricante (grasa grafitada), y que el modo de aplicación era solo en el punzón. Por lo tanto, se realizó un ensayo Pin on Disk para obtener el valor de este coeficiente.

Para la realización de este ensayo se obtuvieron, una muestra de la grasa grafitada como también de la chapa utilizada, siendo esta última cortada en un router CNC, en discos de geometrías normalizadas[6]. Asimismo, se mecanizaron en acero SAE H13 (material del punzón de la máquina de ensayo Erichsen) ocho punzones. Los mismos fueron templados y revenidos a la dureza del punzón real (62HRC).

Finalizada la etapa de preparación, se procedió a realizar el ensayo en una maquina Pin on Disk. En tal sentido se efectuaron cuatro pruebas, mientras en dos de ellas se aplicó lubricación solo en el punzón, en las restantes tanto en el punzón como en la chapa. De los valores obtenidos, se calculó un valor promedio ya que, si bien en el test Erichsen solo se lubrica el punzón, el desplazamiento y estiramiento de la superficie de la chapa, provoca que el lubricante depositado sobre el punzón, se desplace junto con la chapa, habiendo entonces lubricante en ambas superficies.

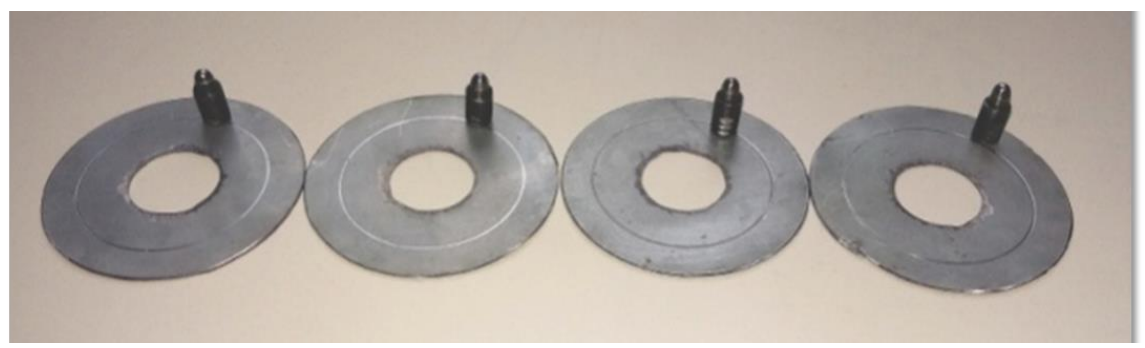

Figura 5: Anillos y punzones utilizados en el ensayo Pin on Disk

También fue necesario definir la velocidad de rotación del anillo seleccionándose un valor final de 
1r.p.s a partir de realizar la correlación entre el diámetro de rotación del punto de apoyo del punzón y su velocidad de descenso durante el ensayo Erichsen, y teniendo en cuenta que esta es la mínima velocidad posible.

Como fuerza normal al disco se utilizó el valor máximo de diseño de la máquina, es decir 19.6N. Hecho esto se procedió a realizar el ensayo Pin on Disk. De cada uno de los cuatro ensayos efectuados se relevaron con un adquisidor de datos, los valores de la fuerza de rozamiento en función del tiempo. Dichos valores fueron exportados a una PC y procesados a fin de obtener el valor del coeficiente de rozamiento correspondiente a cada prueba. Para obtenerlo se aplicó la siguiente fórmula:

$$
\mu=\frac{F r}{19.6} \times 0.979
$$

Siendo Fr la fuerza de rozamiento obtenida con el adquisidor de datos, 19.6 la fuerza normal, y 0.979 un coeficiente en función del brazo de palanca de la máquina. Una vez obtenidos los resultados, se realizaron los gráficos correspondientes al coeficiente de roce por cada prueba:
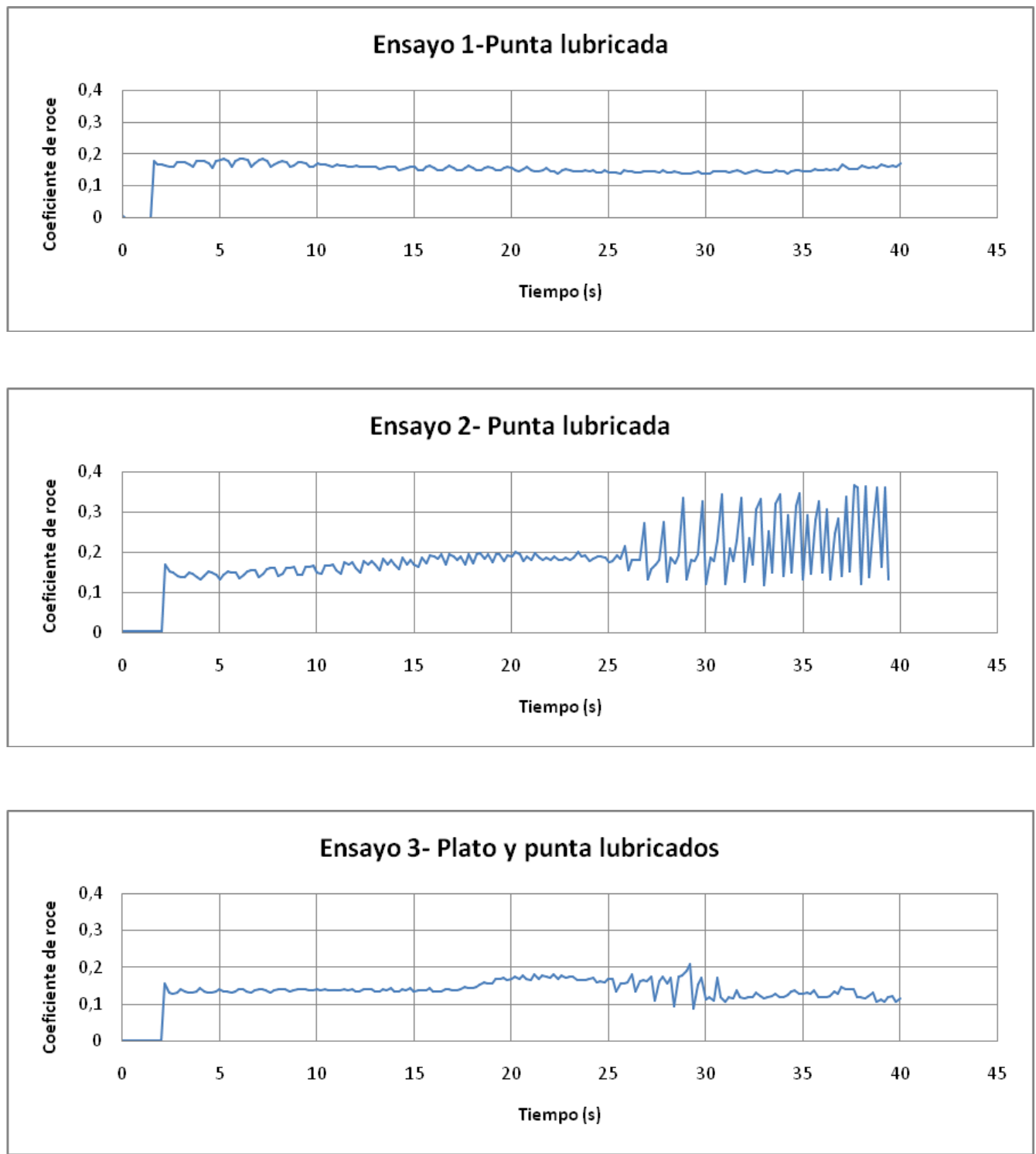


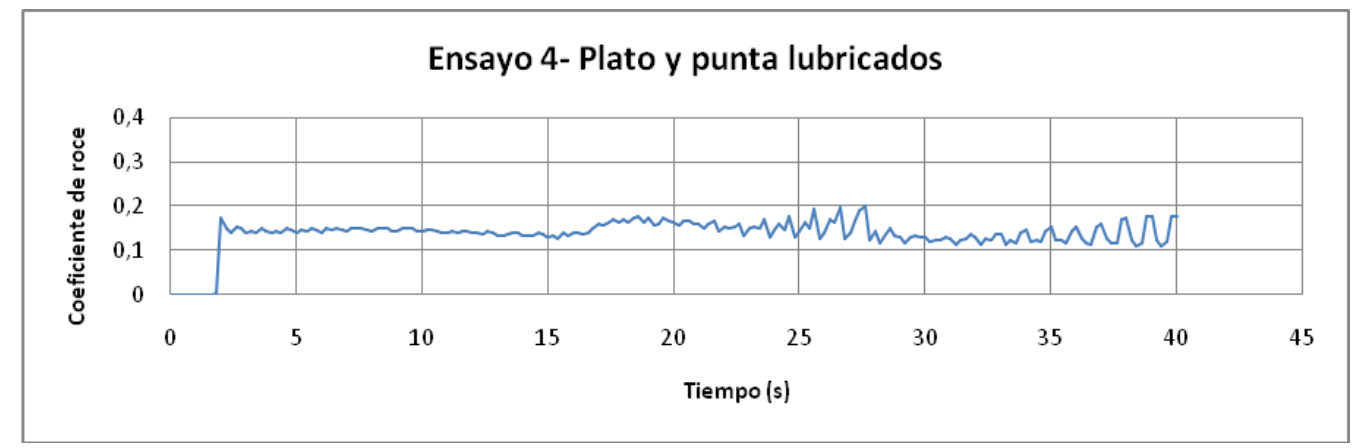

Figura 6: Curvas obtenidas a partir del adquisidor de datos

Se analizaron en forma conjunta las cuatro curvas obtenidas. Eliminando el transitorio inicial, se observa que, durante el lapso comprendido entre los $5 \mathrm{~s}$ y $15 \mathrm{~s}$, los valores alcanzados resultaron estables para todas ellas. En consecuencia, el valor promedio del coeficiente de roce se estimó a partir de estos segmentos, resultando de 0,15 .

Continuando con los parámetros cargados en el simulador, se seleccionó como máquina una prensa hidráulica y se fijó una velocidad de avance de $2 \mathrm{~mm} / \mathrm{s}$.

Otro aspecto importante a considerar es el mallado. En este trabajo, en la chapa se usó una malla constituida de elementos hexaédricos, con un refinamiento de dos grados (ver figura más abajo), aumentando la densidad en la sección limitada por el diámetro interior del prensachapa, donde se desea obtener un importante grado de detalle, ya que según la referencia del ensayo real es allí donde resultará un mayor grado de deformación. El total de elementos de la malla fue de 52083, teniendo en cuenta que se emplearon tres elementos en el espesor. Por otro lado, el resto de las partes involucradas fueron consideradas como sólidas, ya que en ellas no debiera producirse deformación plástica.

Finalmente, en los detalles del proceso de simulación se consideró la recuperación elástica, ya que influye directamente y repercute sobre la medición de espesores y deformación de la pieza real analizada.

\section{RESULTADOS}

Terminado el proceso de simulación, se efectuó una comparación entre los resultados obtenidos respecto a los medidos en la chapa real. En este proceso fueron considerados: la variación de espesor de la chapa en diferentes puntos de la copa, así como el valor máximo de la fuerza del punzón.

En cuanto a la fuerza del punzón, el valor arrojado por el software resultó muy próximo al experimental, $1178.6 \mathrm{~kg}(4 \times 294.649 \mathrm{~kg})$ versus $1250 \mathrm{~kg}$, esto es una diferencia del - 5.7\%.

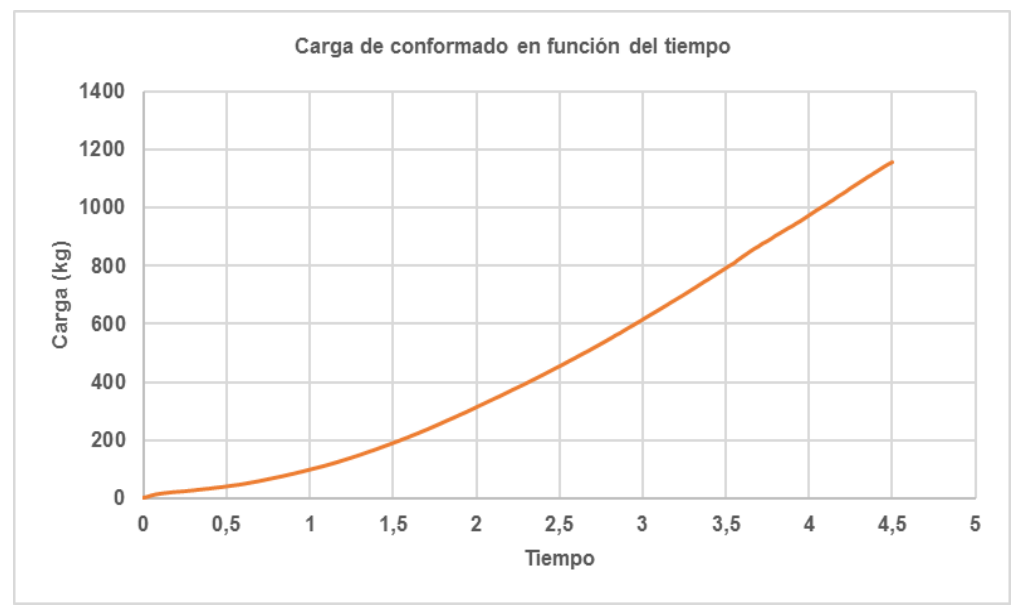

Figura 7: Gráfico de la fuerza del punzón en función del tiempo.

Respecto a los espesores, también resultaron muy similares. Los valores numéricos obtenidos se pre- 
sentan en la tabla 4. De allí surge que la diferencia porcentual promedio es de un 4.1\%. En la figura 13 se visualiza la variación del espesor en la chapa con vista en corte transversal a la dirección de laminación, al igual que el espesor visible en la chapa real cortada.

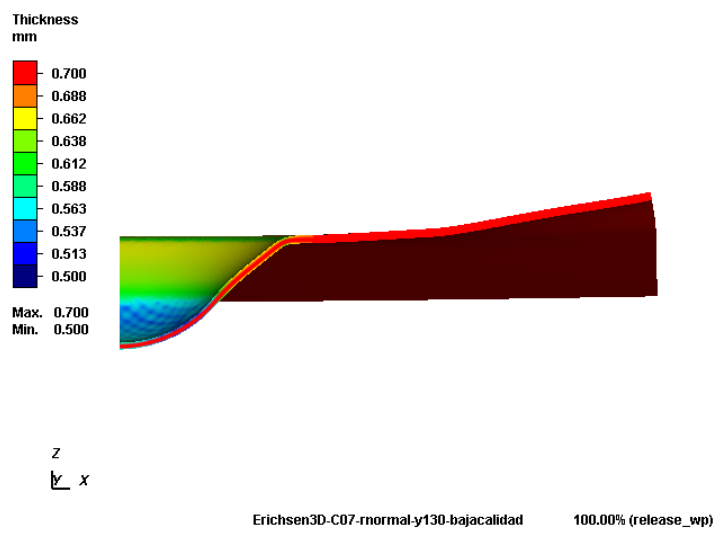

Figura 8: Variación de los espesores en la chapa simulada

Tabla 4: Comparación de espesores reales y simulados

\begin{tabular}{l|l|l|l|l}
\hline ZONA & $\begin{array}{l}\text { VALOR REAL } \\
(\mathrm{MM})\end{array}$ & $\begin{array}{l}\text { VALOR SIMU- } \\
\text { LADO (MM) }\end{array}$ & $\begin{array}{l}\text { DIFERENCIA } \\
(\mathrm{MM})\end{array}$ & $\begin{array}{l}\text { DIFERENCIA } \\
\text { PORCENTUAL } \\
(\%)\end{array}$ \\
\hline $\begin{array}{l}\text { Espesor en altura } \\
\text { máxima }\end{array}$ & 0,59 & 0,57 & $-0,02$ & $-3,4$ \\
\hline $\begin{array}{l}\text { Espesor en altura } \\
\text { media }\end{array}$ & 0,58 & 0,61 & $+0,03$ & $+0,52$ \\
\hline Espesor mínimo & 0,55 & 0,53 & $-0,02$ & $-3,6$ \\
\hline
\end{tabular}

Finalmente, otro detalle a tener en cuenta es el pliegue de la chapa aparecido durante el proceso de estampado en la zona exterior al prensachapa. Esta deformación también aparece en la simulación, y a pesar que no sea un valor cuantitativo, el surgimiento de este defecto indica la representatividad de la simulación. Dicho fenómeno se debe a un proceso de deslizamiento de la chapa hacia el interior de la cavidad de la matriz, combinando así un proceso de estirado con uno de embutido.
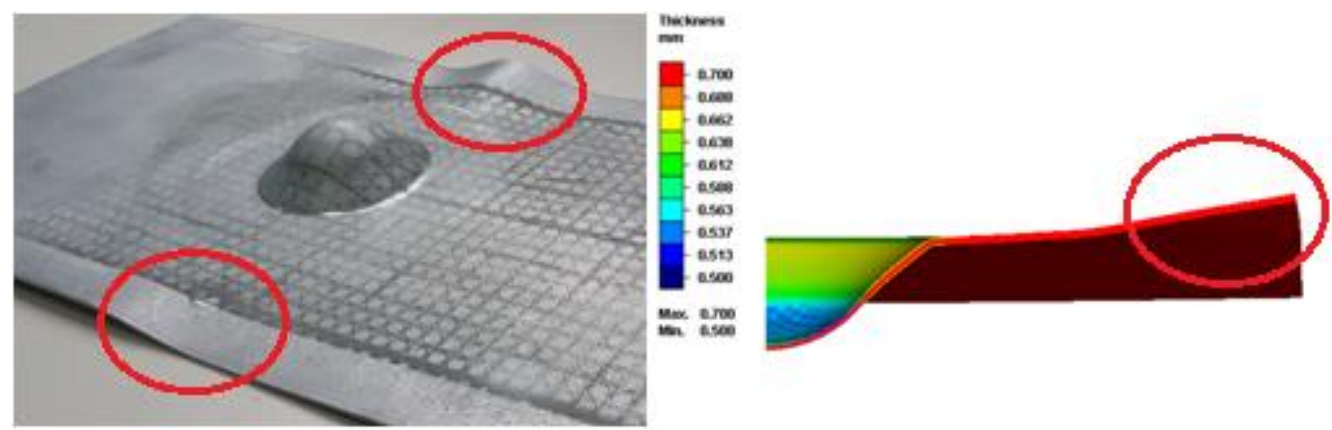

Figura 9: Representación de los pliegues en la simulación 


\section{DISCUSIÓN}

En cuanto a la parametrización del proceso de simulación, la pieza fue sometida a similares condiciones de borde que en el ensayo real. Se realizaron todas las tareas necesarias para lograr el correcto relevamiento numérico de cada uno de estos valores. Respecto al material de la chapa se tuvieron en cuenta las propiedades directamente relacionadas a su composición química, además de introducir los valores de anisotropía, tensión de fluencia y rotura, para que las propiedades relacionadas al proceso de laminación sean también influyentes en el proceso de deformación de la chapa.

Una vez realizada la parametrización se pudo observar una coincidencia entre los valores obtenidos como resultado de la simulación con los medidos en el ensayo físico, sea tanto en la medición de espesores con el banco óptico, como la obtención de la carga del punzón informada por la máquina de ensayos Erichsen.

Es un hecho de remarcar la cantidad de variables dentro del proceso. Esto provoca que el set up para la simulación sea muy laborioso debido al número de parámetros y a la dificultad de obtener el valor cuantitativo de algunos de ellos, como es el caso del coeficiente de fricción.

Para lograr una clara visualización del desvío entre los valores reales y simulados en cada uno de los parámetros analizados, se confeccionó un gráfico donde se le asignó el equivalente al $100 \%$ al resultado numérico medido en el ensayo físico y su correspondiente porcentual a los valores obtenidos por simulación. A continuación, se adjunta dicho gráfico.

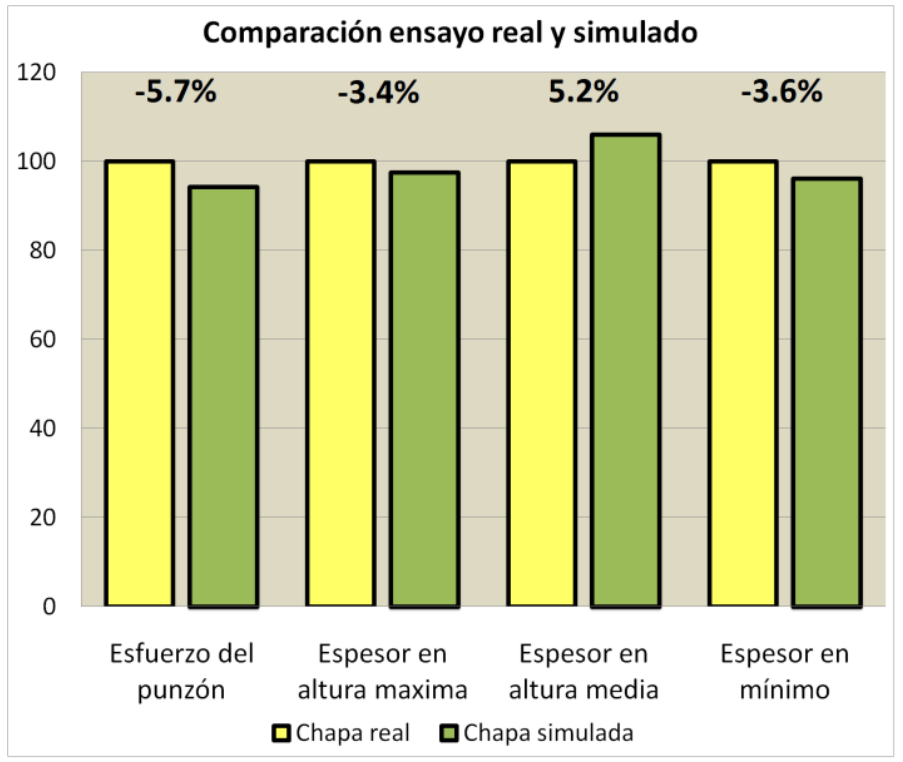

Figura 10: Comparación de mediciones realizadas en la chapa real y la simulada

\section{CONCLUSIONES}

- El valor obtenido para el esfuerzo del punzón presentó una diferencia del - 5.7\% respecto del ensayo real.

- En cuanto a los espesores, los obtenidos en la simulación, varían en una media del $4.1 \%$.

- Simufact.Forming no sólo recreó la copa producida por la penetración del punzón, sino también los pliegues formados en la chapa, en la zona exterior al diámetro del prensachapa.

\section{BIBLIOGRAFÍA}

[1] GAVRUS, A., "An inverse analysis approach of the Erichsen test starting from a finite element model", International Journal of material forming, v. 1, pp. 5-8, 2008. 
[2] OLEKSIK, V. "Experimental and finite element analysis of Erichsen test. Application to identification of sheet metallic material behavior", Galati, fascicle 5, pp. 81-86, 2009.

[3] AKROUT, M., y otros, "Numerical and experimental study of the Erichsen test for metal stamping", Advances in Production Engineering \& Management, 3, pp. 81-92, 2008.

[4] ESMAEILIZADEH, R., y otros, "Simulated and experimental investigation of stretch sheet forming of commercial AA1200 aluminum alloy", Transactions of nonferrous metals society of China, 24, pp. 484-490, 2014.

[5] NORMA UNE-EN 10130:2008, "Productos planos laminados en frío de acero bajo en carbono para embutición o conformación en frío. Condiciones técnicas de suministro”, May. 2008.

[6] NORMA ASTM G9-05, "Standard Test Method for Wear Testing with a Pin-on-Disk Apparatus", 2010. 